\section{CARE AND USE OF MICROWAVE OVEN FOR DIAGNOSTIC PATHOLOGY}

M.A. Hayat, Kean University, Union, NJ mhayat@turbo.kean.edu

It is well known that biopsy, surgical and postmortem tissues fixed in formalin and embedded in paraffin require antigen retrieval for diagnostic pathology. Various methods, including heating, enzymatic digestion, ultrasonication, and detergents, are in use for achieving antigen retrieval. A combination of these methods is also employed. However, the most effective methodology for antigen retrieval in a wide variety of tissues involves heating at high temperature using a microwave oven, an autoclave, a pressure cooker, or a hot plate. Among these treatments, heating the tissue sections ( $4 \mu \mathrm{m}$ thick) in $0.01 \mathrm{M}$ sodium citrate buffer $(\mathrm{pH} 6.0)$ in a microwave oven is the most widely used technique for antigen retrieval. To obtain efficient and reproducible antigen retrieval, the microwave oven must be used carefully and optimally. The following steps are recommended to achieve these goals (Hayat, $2000 \mathrm{a}, \mathrm{b}$ ).

The causes of most hazards encountered in using a microwave oven are straight forward and can be avoided by taking necessary precautions. Higher power settings and longer durations of heating than optimal for a given study should be avoided. Because overheating is not uncommon, the time setting should be checked. The fluid contents of the container heat faster than the container. In fact, the fluid contents of the container heat so fast that the container can still be cool. Even after the container has been removed from the oven, the container will become hotter for a period of time. Changes in the size, shape, and nature of the container and its position in the microwave oven result in significant changes in the temperature of the container fluid. Furthermore, changes of these factors will change the temperature of the container fluid even if the volume of the container contents remains unchanged.

Overheating the microwave oven tends to result in boiling or excessively rapid evaporation of fluids such as ethanol used for dehydration, formaldehyde employed for fixation, and the antigen retrieval fluid. As a result flammable and/or toxic materials are released in the microwave oven. Even without overheating, vapors are produced because containers are kept open in the oven to prevent pressurization. Microwave transparent containers should be used. It is preferred to use fluid volumes of $\sim 100 \mathrm{ml}$. Microwave ovens with a temperature probe are also available. To avoid possible exposure to toxic vapors, the face should be turned away when the oven door is opened. Oven door should not be opened or closed to turn the microwave power on and off.

Areas of high microwave flux should be checked, using a Pelco 36140 microwave bulb array (Ted Pella, Reading, CA). Specimens should not be placed in such areas which are indicated by illuminated bulbs. Vials containing the specimens should be placed in a water bath $(50 \mathrm{ml})$ that has been preheated to the required temperature. The temperature should be regulated by placing microwave temperature probe into a vial of the same solution that is present in the specimen vial. The built-in temperature probe displays the temperature on the oven front panel. The wire that attaches the probe to the oven should be submerged in the water to decrease the antennae effect. An additional $400 \mathrm{ml}$ of static water load should be placed in the oven at an optimal position determined with the microwave bulb array. This water is changed between every step.
The following specific steps must be taken while using a microwave oven for antigen retrieval.

1. Test microwave leakage with a microwave detector with a low sensitivity range.

2. Place the oven in an efficient fumehood.

3. Wear gloves while using your hands inside an oven.

4. Predetermine the maximum power level of the oven used.

5. Do not place a container with a closed lid in the oven.

6. Do not use high temperature settings unless absolutely necessary.

7. Predetermine the irradiation time, and check the time setting

8. Check the actual temperature attained by the specimen.

9. Predetermine the amount of specimens to be irradiated.

10. Predetermine the exact position of the specimen in the oven during irradiation.

11. Predetermine the amount of water load and its place in the oven.

12. Find out the extent of hot spots in the oven.

13. Use Teflon containers with thick walls in the oven. Plastic containers can also be used.

14. Do not use metals or foils in the oven.

15. Contrary to some published reports, pencil-written materials can be used in the oven.

Hayat, M.A. 2000a. Principles and Techniques of Electron Microscopy: Biological Applications, 4th ed. Cambridge University Press, New York and Cambridge.

Hayat, M.A. 2000b. Antigen Retrieval Methods and Microwave Heating. Springer-Verlag, New York and Berlin.

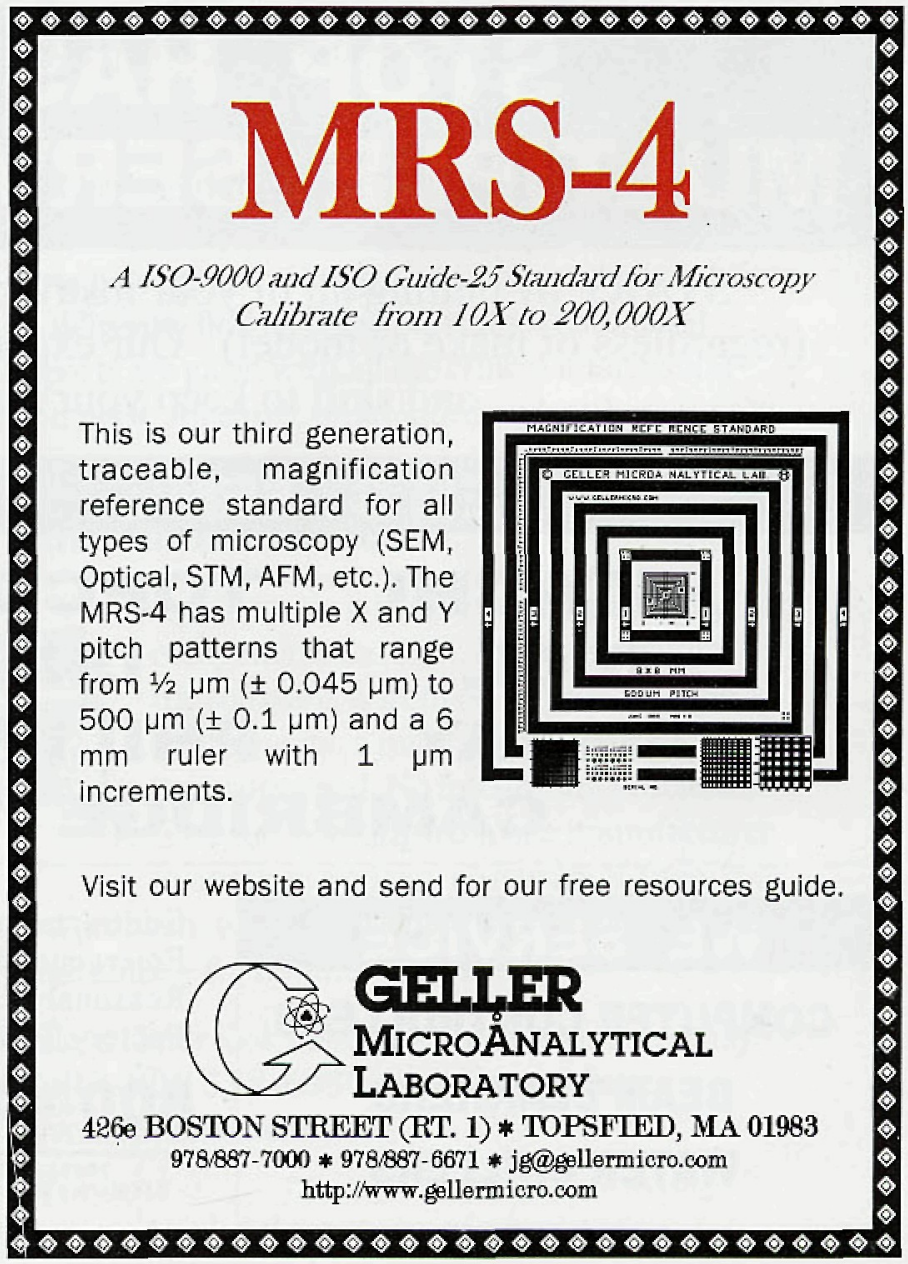

